\title{
Editorial
}

We are pleased to launch this new journal in the rapidly developing field of laser chemistry. It is our intent to bridge the gap between physics and chemistry laser-related research with contributions by scientists working in both disciplines. The journal will, for the first time, unite worldwide laser chemistry research activities in one publication. Laser Chemistry will cover a wide range of topics but with particular focus on the following:

\section{Laser-selective chemistry}

Reactive scattering and state-to-state dynamics

Multiphoton dissociation, ionization and absorption phenomena

Laser-assisted collisions and reactions in bulks and on surfaces

Primary processes in photochemistry and chemiluminescence

Molecular relaxation processes: dephasing, energy redistribution and ultrafast dynamics

New laser techniques

Applications of lasers in biology

We welcome original theoretical and applied papers, as well as overviews. In addition, the journal will publish rapid communications, which may be submitted in camera-ready copy form, after the refereeing process, for speed of publication. Special instructions to authors for preparation of camera-ready typescripts will be supplied by any one of the editors. Papers should be submitted directly to the editor at the nearest geographic location.

We hope that the journal's commitment to scientific excellence will help contribute to exciting developments in laser chemistry throughout the world.

The Editors 